\title{
La bioluminiscencia como herramienta para evaluar el lavado de manos durante la formación de profesionales relacionadas con la Salud Pública
}

\author{
Bioluminescence as a tool to evaluate hand washing during the training of \\ professionals related to Public Health \\ Sara Contreras', Gustavo Caro ${ }^{2}$, Jocelyn Cuevas², Carlos Barrientos ${ }^{2}$, \\ Alvaro Opazo ${ }^{3,4}$
}

\section{Resumen}

Las Infecciones Asociadas a Atención en Salud (IAAS), las Enfermedades Transmitidas por Alimentos (ETAS) y las zoonosis generan altos costos a la salud pública. Una medida sencilla y económica, y con evidencia de su efectividad para prevenirlas es el lavado de manos, protocolo establecido por la Organización Mundial de la Salud (OMS), práctica que se enseña en los programas de formación profesional a estudiantes de la salud humana y animal, así como a quienes procesan alimentos. El objetivo de este estudio descriptivo y transversal fue evaluar el lavado de manos mediante bioluminiscencia en estudiantes durante su desempeño en los centros de práctica, así como su conocimiento y actitud hacia el lavado de mano, mediante una encuesta a 45 estudiantes de las carreras de Gastronomía, Enfermería y Medicina Veterinaria en su último ciclo de formación académica de una institución de Educación Superior Privada (Concepción, Chile). Los estudiantes presentan un adecuado conocimiento sobre el lavado de mano (90\%), pero una mala actitud frente a este $(70 \%$ de respuestas favorables). Los estudiantes le dan mayor relevancia al lavado de mano cuando son observados, evaluados o cuando sus compañeros lo realizan, mostrando una falta de conciencia respecto a la importancia de realizarlo. La prueba de bioluminiscencia indicó una reducción significativa entre

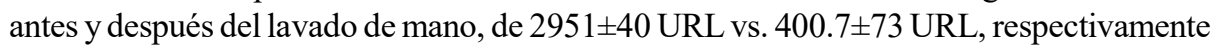

\footnotetext{
${ }^{1}$ Universidad Andres Bello, Escuela de Enfermería, Facultad de Enfermería, Concepción, Chile

${ }^{2}$ Escuela de Medicina Veterinaria, Facultad de Recursos Naturales y Medicina Veterinaria, Universidad Santo Tomás, Chile

${ }^{3}$ Universidad Andres Bello, Escuela de Medicina Veterinaria, Facultad de Ciencias de la Vida, Concepción, Chile

${ }^{4}$ E-mail: alvaro.opazo@unab.cl
}

Recibido: 4 de noviembre de 2019

Aceptado para publicación: 20 de junio de 2020

Publicado: 11 de agosto de 2020 
$(\mathrm{p}<0.0001)$, sin diferencias significativas entre las carreras. La medición de ATP mediante bioluminiscencia es un método sencillo y rápido para evaluar la eficacia del lavado de mano, siendo una herramienta que puede incorporarse dentro de la formación disciplinar en carreras directamente relacionadas con la salud humana-animal.

Palabras clave: higiene de manos, ATP, actitud, conocimientos

\title{
AbSTRaCT
}

\begin{abstract}
Health Care Associated Infections (HAS), Foodborne Illness and zoonoses generate high costs for public health. A simple and inexpensive measure, and with evidence of its effectiveness in their prevention is handwashing, a protocol established by the World Health Organization (WHO), that is taught in professional training programs to students of human and animal health, as well as those who process food. The aim of this descriptive and cross-sectional study was to evaluate handwashing by bioluminescence in students during their performance in practice centres, as well as their knowledge and attitude towards handwashing, by a survey involving 45 students from the Gastronomy, Nursing and Veterinary Medicine in their last cycle of academic training of a private higher education institution (Concepción, Chile). The students showed an adequate knowledge of handwashing (90\%), but a bad attitude towards it ( $70 \%$ of favourable responses). Students gave greater importance to handwashing when they are observed, evaluated or when their classmates do it, showing a lack of awareness regarding the importance of doing it. The bioluminescence test indicated a significant reduction between before and after

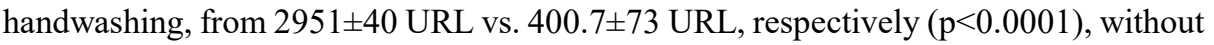
significant differences between the careers. The measurement of ATP by bioluminescence is a simple and rapid method to evaluate the effectiveness of handwashing, being a tool that can be incorporated into disciplinary training in careers directly related to humananimal health.
\end{abstract}

Key words: hand washing, ATP, attitude, knowledge

\section{INTRODUCCIÓN}

La salud pública a nivel mundial se enfrenta a dos grandes problemas, las Infecciones Asociadas a Atención en Salud (IAAS) y las Enfermedades Transmitidas por Alimentos (ETA). Se estima que las IAAS afectan a un $10 \%$ de los pacientes hospitalizados, con un aumento de la morbilidad en aproximadamente 600 millones de habitantes, mientras que las ETA producen una mortalidad cercana a 420 mil personas (OMS, 2005). Ambas conllevan altos costos para el sistema de salud público, producto del aumento en los días de hospitalización ( 1 a 25 días adicionales o entre US\$ 600 a 40,000/episodio), dependiendo del tipo de infección y de los exámenes y procedimientos complementarios que se realizan (Weinstein et al., 2008).

En respuesta a esta alarmante situación, la Organización Mundial de la Salud (OMS) en 2005 sugirió y propuso la Higiene de Manos, como una medida de prevención senci1la, rápida y económica, para la prevención de las IAAS y ETA, que consiste en la higienización con alcohol gel y el lavado de manos, la cual disminuye los riesgos de infecciones (OMS, 2005). El lavado o higiene de manos es una técnica que se enseña desde la primera infancia en los hogares y luego en la 
educación primaria en Chile (Unidad de Curriculum y Evaluación del Ministerio de Educación de Chile - https://www.curriculumnacional.cl/614/w3-article-32881.html\#tabs_3) como en otros países, y luego curricularmente en carreras profesionales del área de la Salud, como en Medicina, Enfermería, Obstetricia, Medicina Veterinaria, Técnico o Auxiliar veterinario, y en aquellas relacionadas con la manipulación de alimentos como Gastronomía.

En estas carreras profesionales el uso de las manos es fundamental, y estas son consideradas vehículos transmisores de microorganismos patógenos, ya que la piel presenta una flora saprófita que puede ser contaminante y fuente de infecciones nosocomiales (Pittet et al., 2006; Rey y Prieto, 2015; Nante et al., 2017). Ante esto, la Organización Mundial de la Salud (OMS), en su documento «Directrices de la OMS sobre higiene de las manos en atención sanitaria» (OMS, 2005) pone en marcha la campaña «Salve vidas: límpiese las manos», destinada a mejorar las prácticas de higiene de manos del personal sanitario a nivel mundial (Morán Cortés et al., 2014), utilizando como pilar la formación, aplicación e investigación, en materia de seguridad del paciente. Por su parte, la FAO junto con la OMS, crean el Codex Alimentarius con el objetivo de promover la higiene alimentaria en los profesionales que elaboran, manipulan y procesan alimentos (WHO, 2013).

En Medicina Veterinaria no existen indicaciones propias, y se debe seguir el protocolo de higiene de manos propuesta por la OMS; sin embargo, su aplicación se acota principalmente a las buenas prácticas de la asepsia quirúrgica y la forma correcta del lavado de manos, incluidos en la literatura quirúrgica (Fossum, 2013). No obstante, y debido a que entre el 60 y $76 \%$ de los brotes de enfermedades infecciosas emergentes son zoonosis (Lloyd-Smith et al., 2009), es que la higiene de manos ha tomado mayor importancia en estos profesionales, al estar en con- tacto directo con secreciones, sangre o desechos corporales de animales, quedando más expuestos a patologías y ser transmisores de ellas.

La bioluminiscencia es una herramienta de apoyo inmediato que detecta la adenosina trifosfato (ATP) presente en restos de materia orgánica, bacterias, hongos y otros microorganismos, pudiéndose medir los niveles de contaminación en superficies y muestras de agua, como también, evaluar los procedimientos de limpieza e higienización, en superficies, alimentos e higiene de manos (Seeger y Griffiths, 1994; Nante et al., 2017), mediante instrumental especializado, permitiendo implementar soluciones inmediatas en caso de obtener resultados inaceptables de contaminación, como también demostrar si el procedimiento de higienización fue adecuado (Pittet et al., 2006). Sin embargo, aún existen problemas en la adherencia a esta sencilla práctica (Borges Primo et al., 2010), de allí que el objetivo del presente estudio fue determinar la eficacia y el grado de conocimiento posterior al lavado de manos en estudiantes de Enfermería, Medicina Veterinaria y Gastronomía en una institución de educación superior.

\section{Materiales y Métodos}

\section{Lugar de Muestreo}

El estudio se llevó a cabo en los centros de práctica de la carrera de Gastronomía, Medicina Veterinaria y de Enfermería de una institución de educación superior privada ubicada en la provincia de Concepción, región del Biobío, Chile.

\section{Tamaño Muestral}

El tamaño muestral corresponde a 45 estudiantes, agrupados en: 15 estudiantes del taller de cocina de la carrera de Gastronomía; 15 estudiantes de la carrera de Medicina Veterinaria; y 15 estudiantes de la carrera 
de Enfermería. Los participantes fueron seleccionados mediante un muestreo por conveniencia, debido a que se seleccionó el tamaño de muestra de forma directa e intencional, seleccionando a estudiantes que cursaban sus prácticas profesionales y que debieron recibir durante su formación profesional la importancia del lavado o higiene de manos, al formar parte de las competencias curriculares que deben adquirir durante su formación.

\section{Procedimientos}

Cada uno de los participantes dieron su conformidad de ingresar al estudio firmando el formulario de consentimiento informado, estudio que fue previamente autorizado por el Comité de Ética de la institución educativa bajo los códigos 039-16 y 040-16.

En una reunión inicial se les explicó el objetivo y procedimientos a realizar. Se procedió a la toma de consentimiento informado y se les dijo que los investigadores llegarían a sus lugares de práctica de manera sorpresiva. La persona a cargo de esta investigación, sin anunciarse, ingresaba a los recintos de práctica, y se les pedía que dejaran de hacer lo que estaban realizando y dispusieran sus manos al descubierto para poder tomar la muestra por la superficie de las manos usando una tórula $3 \mathrm{M}{ }^{\circledR}$ Clean-Trace ${ }^{\mathrm{TM}}$ UXL-100 $(3 \mathrm{M} ®$ Chile) de tapa de color azul, siguiendo el protocolo del fabricante. Luego se les solicitaba que realizaran un lavado de manos de la manera en que se realiza en sus centros de práctica y según el protocolo que se les haya instruido durante su formación. Seguido a esto, se volvió a tomar la muestra con una tórula nueva.

Las tórulas, después de ser pasadas por las manos de los estudiantes era introducidas en el equipo $3 \mathrm{M}{ }^{\circledR}$ Clean-Trace ${ }^{\mathrm{TM}}$ Luminómetro $(3 \mathrm{M} \AA)$ que, en el transcurso de 10 segundos entrega un resultado expresado en unidades relativas de luz (URL). Para ello se definió como «aceptado» un valor de $<500$ URL (Lewis et al., 2008). Es importante mencionar que el mismo investigador tomó todas las muestras con el fin de estandarizar el protocolo de muestreo en manos.

Además, cada uno de los 45 estudiantes respondieron el «Cuestionario de lavado de manos y Desinfección» (Anexo 1) adaptado para este estudio con el fin de poder determinar el nivel de conocimiento y actitudes sobre el lavado de manos. Este cuestionario fue adaptado del cuestionario publicado por González-Cabrera et al. (2012) «Cuestionario para evaluar en médicos conductas, conocimientos y actitudes sobre la higiene de manos», que evalúa el conocimiento y actitudes sobre el lavado de manos en médicos. No obstante, para mantener la validez de este cuestionario se sometió a una nueva validación, según describe Caparó (2016) obteniendo un alfa de Cronbach de 0.81. El cuestionario aplicado posee dos partes, la primera con información sociodemográficas y la segunda con aspectos importantes para la higiene de manos.

\section{Análisis Estadístico}

El estudio es de tipo descriptivo y transversal. Los resultados fueron analizados estadísticamente en el programa SPSS 20.0 utilizando la prueba de Kolmogorov-Smirnov para determinar la distribución normal de los datos, y luego la aplicación de la prueba $\mathrm{T}$ o de Wilcoxon (pareada no paramétrica) para determinar si existe diferencia estadísticamente significativa entre el valor URL prey pos-lavado de manos. Luego se obtuvo la diferencia entre pos-lavado según la carrera para someterlos a una prueba U Mann Whitney (no pareada no paramétrica) con el fin de determinar si existen diferencias entre los tres grupos de estudiantes que representan las tres carreras. El nivel de significancia quedó determinado en un valor $\mathrm{p}<0.05$. 


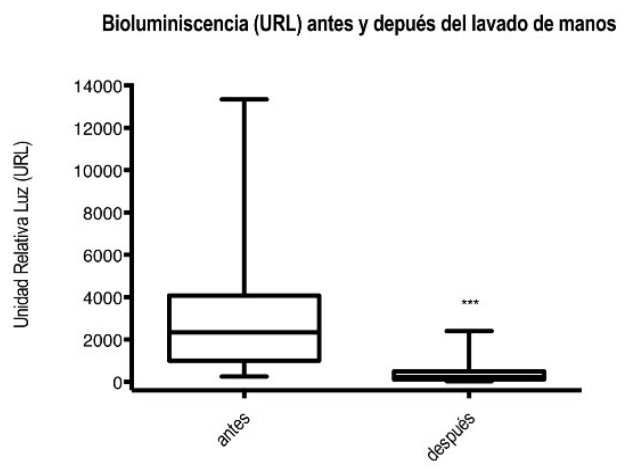

Figura 1. Cajas de bigotes comparando los resultados de la bioluminiscencia en 45 estudiantes durante las actividades disciplinares en los centros de práctica, antes y después del lavado de manos. $* * * \mathrm{p}<0.001$

\section{Resultados}

\section{Unidad Relativa de Luz Obtenidos An- tes y Después del Lavado de Manos}

De las variables sociodemográficas registradas, el 64\% (29) de los estudiantes fueron mujeres y $36 \%$ (16) hombres, con una edad promedio de 25.5 años y un rango de edad entre 19 y 44 años.

El promedio obtenido a través de la bioluminiscencia fue de $2951 \pm 405$ URL antes del lavado de manos y de $400.7 \pm 73.9$ URL después del lavado de manos $(\mathrm{p}<0.0001$; Wilcoxon datos pareados). Sin embargo, y como se observa en la Figura 1, la dispersión de los valores obtenidos en ambas mediciones es amplia, destacándose el valor máximo de 13343 URL antes del lavado de manos, comparado al valor máximo obtenido posterior al lavado de manos de 2401 URL.

Solo el 6\% (3 de 45 estudiantes) obtuvieron un valor menor a 500 URL antes del lavado de manos, valor considerado como adecuado de higienización de las manos; sin embargo, luego del lavado de manos la pro- porción de estudiantes con valores menores a 500 URL subió a 75\% (34 individuos). No obstante, $25 \%$ (11) de los estudiantes no tuvieron una correcta higienización de sus manos posterior al lavado.

El análisis de los resultados antes vs después del lavado de manos indica diferencia significativa $(\mathrm{p}<0.001$, Wilcoxon para muestras pareadas, no paramétrica) para cada una de las carreras (Figura 2). No obstante, se aprecian alarmantes resultados URL antes del lavado de manos con distintas dispersiones en sus valores, con un promedio para las tres carreras que supera en cinco veces el valor cohorte de 500 URL (Cuadro 1). De manera particular, llama la atención los valores máximos de 13343 URL obtenido en la carrera de Enfermería, seguido por Gastronomía con 10392 URL y por Medicina Veterinaria con 8016 URL. Sin embargo, una vez que los estudiantes se lavaron las manos con la técnica que aprendieron durante su formación académica y con los medios disponibles al momento del muestreo en el centro de prácticas utilizando jabón cosmético, se observa como los promedios de URL disminuyeron de manera significativa $(\mathrm{p}<0.001)$ en los estudiantes de las tres carreras. Se observa, además, que en Medicina Veterinaria los resultados son menos dispersos, seguido de Gastronomía y Enfermería (Cuadro 1). Por otro lado, llama la atención que el valor URL promedio para Gastronomía posterior al lavado de manos se mantuvo por encima del valor cohorte esperado (541.7 vs 500 URL).

\section{Análisis del Cuestionario Sobre Conoci- mientos y Actitud hacia el Lavado de Ma- nos}

En el Cuadro 2 se observa las respuestas dicotómicas expresadas en frecuencia relativa y absoluta para las preguntas 3 a 10 del cuestionario, que miden el conocimiento frente a la higiene de mano. En la pregunta 3, $¿$ Ha recibido información sobre la higiene de manos durante su formación académica?, 7\% 


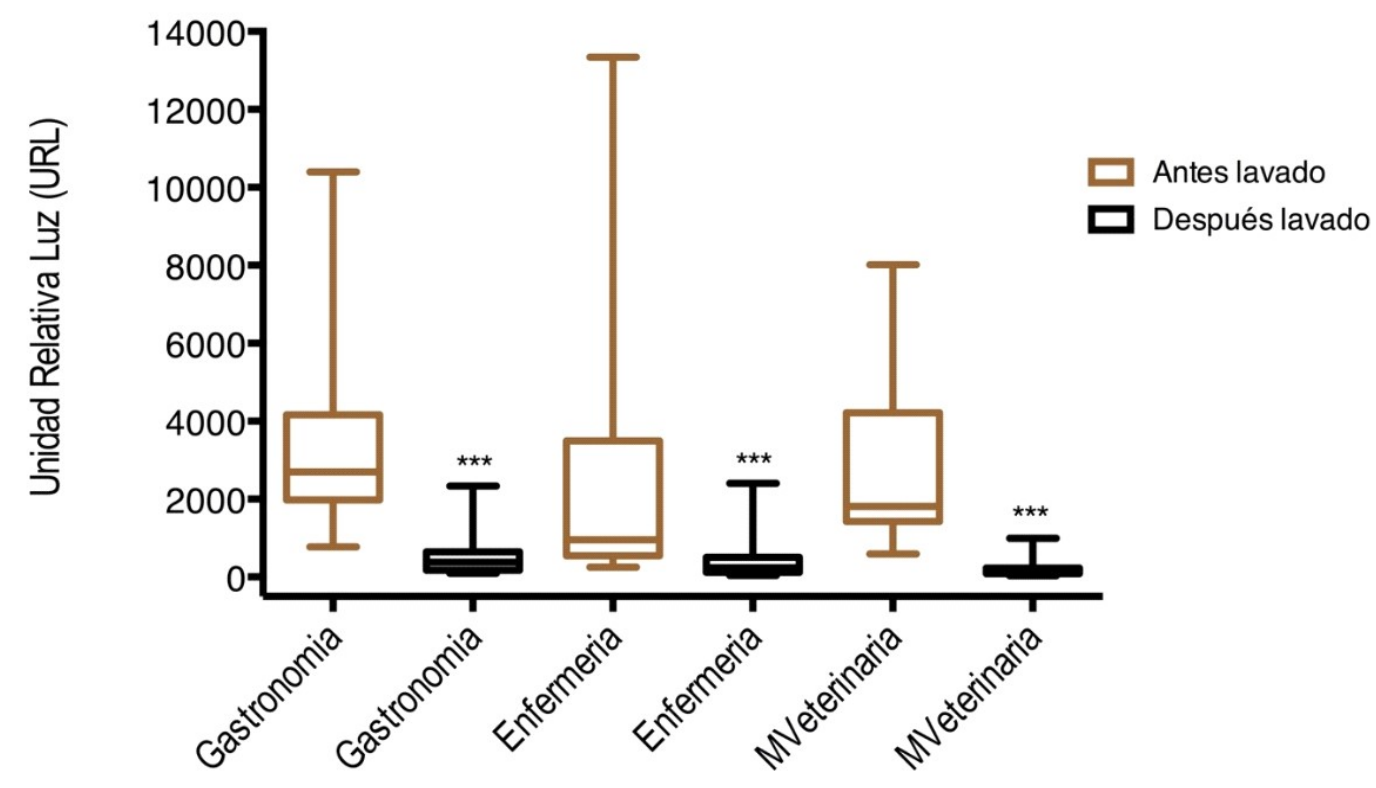

Figura 2. Cajas de bigotes que permite comparar los resultados de la medición mediante bioluminiscencia, antes y después del lavado de manos ( $\mathrm{n}=15$ estudiantes por carrera). ${ }^{* * *}: \mathrm{p}<0.001$

Cuadro 1. Estadística descriptiva de los resultados de URL (unidades relativas de luz) obtenidos antes y después del lavado de manos, según la carrera de los estudiantes $(\mathrm{n}=15$ por carrera)

\begin{tabular}{lcccccc}
\hline & \multicolumn{2}{c}{ Gastronomía } & \multicolumn{2}{c}{ Enfermería } & \multicolumn{2}{c}{$\begin{array}{c}\text { Medicina } \\
\text { Veterinaria }\end{array}$} \\
\cline { 2 - 7 } & Antes & Después & Antes & Después & Antes & Después \\
\hline Promedio & 3,557 & 541.7 & 2,589 & 432.5 & 2,708 & 227.9 \\
Error estándar de & 684.1 & 141.1 & 874.3 & 151.9 & 523.9 & 69.9 \\
la media & & & & & & \\
Mínimo & 770 & 94 & 254 & 38 & 592 & 32 \\
Máximo & 10,392 & 2,330 & 13,343 & 2,401 & 8,016 & 996 \\
Percentil 25\% & 1,982 & 177 & 548 & 120 & 1,430 & 88 \\
Percentil 75\% & 4,164 & 646 & 3,488 & 508 & 4,218 & 226 \\
Límite inferior & 2,090 & 239.1 & 713.3 & 106.6 & 1,584 & 78.01 \\
IC 95\% & & & & & & \\
Límite superior & 5,024 & 844.3 & 4,464 & 758.4 & 3,832 & 377.9 \\
IC 95\% & & & & & & \\
\hline
\end{tabular}


Cuadro 2. Resultados de las preguntas 3 a 10 del cuestionario, que tienen relación con el conocimiento frente a la higiene de manos de los encuestados $(n=45$ estudiantes de las carreras de Gastronomía, Enfermería y Medicina Veterinaria)

\begin{tabular}{lcccc}
\hline & \multicolumn{2}{c}{ Sí } & \multicolumn{2}{c}{ No } \\
\cline { 2 - 6 } Pregunta & $\%$ & $\mathrm{n}$ & $\%$ & $\mathrm{n}$ \\
\hline $\begin{array}{l}\text { 3. ¿Ha recibido usted formación específica sobre } \\
\text { higiene de manos? }\end{array}$ & 93 & 42 & 7 & 3 \\
$\begin{array}{l}\text { 4. La información sobre higiene de manos que ha } \\
\text { recibido es la adecuada }\end{array}$ & 91 & 41 & 9 & 4 \\
$\begin{array}{l}\text { 5. ¿Cree usted haber sido, de forma inconsciente, } \\
\text { un medio de transmisión de enfermedades en } \\
\text { alguna ocasión? }\end{array}$ & 56 & 25 & 44 & 20 \\
$\begin{array}{l}\text { 6. La mejor opción para la higiene de manos es el } \\
\text { agua y el jabón }\end{array}$ & 82 & 37 & 18 & 8 \\
$\begin{array}{l}\text { 7. Las uñas largas aumentan el riesgo de } \\
\text { transmisión de infecciones }\end{array}$ & 100 & 45 & 0 & 0 \\
$\begin{array}{l}\text { 8. Debe realizarse un lavado de manos cada vez que } \\
\text { iniciamos un turno de trabajo }\end{array}$ & 100 & 45 & 0 & 0 \\
$\begin{array}{l}\text { 9. Cuando se utilizan guantes puede prescindirse de } \\
\text { la higiene de manos }\end{array}$ & 18 & 8 & 82 & 37 \\
$\begin{array}{l}\text { 10.Llevar anillos y/o pulseras aumenta la } \\
\text { probabilidad de contaminación de las manos }\end{array}$ & 100 & 45 & 0 & 0 \\
\hline
\end{tabular}

de los estudiantes manifestó no haber recibido información durante sus 10 semestres de formación profesional, todos ellos de la carrera de Medicina Veterinaria, a diferencia de las otras dos carreras que todos indicaron que recibieron la información respectiva $(\mathrm{p}=0.04)$. En forma similar, en la pregunta 4 ¿La información sobre higiene de manos que ha recibido es la adecuada? El 9\% responde negativamente, siendo todos ellos de la carrera de Medicina Veterinaria, a diferencia con los estudiantes de las otras dos carreras que todos consideraron adecuada la formación recibida $(\mathrm{p}=0.012)$.
Conociendo la relevancia del lavado de manos como estrategia para bloquear la cadena de transmisión de patógenos, la pregunta 5, ¿Cree usted haber sido, de forma inconsciente, un medio de transmisión de enfermedades en alguna ocasión?, el 55.6\% reconoce esta situación, siendo los estudiantes de Enfermería los más conscientes con $66.7 \%$, seguidos por los de Medicina Veterinaria $(53.3 \%)$ y finalmente Gastronomía $(46.7 \%)(\mathrm{p}>0.05)$.

A la pregunta 6, ¿La mejor opción para la higiene de manos es el agua y el jabón?,- 
Cuadro 3. Resultados de las preguntas 3 a 10 del cuestionario, que tienen relación con la actitud frente a la higiene de manos de los encuestados ( $\mathrm{n}=45$ estudiantes de las carreras de Gastronomía, Enfermería y Medicina Veterinaria)

\begin{tabular}{|c|c|c|c|c|}
\hline \multirow{2}{*}{ Pregunta } & \multicolumn{2}{|c|}{ Sí } & \multicolumn{2}{|c|}{ No } \\
\hline & $\%$ & $\mathrm{n}$ & $\%$ & $\mathrm{n}$ \\
\hline $\begin{array}{l}\text { 3. Las personas se ven perjudicadas al no lavarme } \\
\text { las manos en mi trabajo }\end{array}$ & 96 & 43 & 4 & 2 \\
\hline $\begin{array}{l}\text { 4. Realizaría higiene de manos con más frecuencia } \\
\text { si mi superior/a lo hiciera cuando vamos a } \\
\text { empezar una actividad }\end{array}$ & 53 & 24 & 47 & 21 \\
\hline $\begin{array}{l}\text { 5. Realizaría higiene de manos con más frecuencia } \\
\text { si mis compañeros lo hicieran }\end{array}$ & 42 & 19 & 58 & 26 \\
\hline $\begin{array}{l}\text { 6. Realizaría higiene de manos con más frecuencia } \\
\text { si no hacerlo perjudicara a las personas. }\end{array}$ & 78 & 35 & 22 & 10 \\
\hline $\begin{array}{l}\text { 7. Realizaría higiene de manos con más frecuencia } \\
\text { si verdaderamente fuera tan importante }\end{array}$ & 78 & 35 & 22 & 10 \\
\hline $\begin{array}{l}\text { 8. Realizaría higiene de manos con más frecuencia } \\
\text { si mis compañeros/as me llamaran la atención } \\
\text { por no hacerlo }\end{array}$ & 71 & 32 & 29 & 13 \\
\hline $\begin{array}{l}\text { 9. Realizaría higiene de manos con más frecuencia } \\
\text { si mi comportamiento estuviera siendo } \\
\text { observado y/o registrado por el supervisor }\end{array}$ & 60 & 27 & 40 & 18 \\
\hline $\begin{array}{l}\text { 10. Realizaría higiene de manos con más frecuencia } \\
\text { si me dejaran tiempo para hacerlo }\end{array}$ & 73 & 33 & 27 & 12 \\
\hline $\begin{array}{l}\text { 11. Transmito la importancia de la Higiene de } \\
\text { Manos a mis compañeros/as y/o a estudiantes de } \\
\text { otros cursos }\end{array}$ & 87 & 39 & 13 & 6 \\
\hline
\end{tabular}

solo $82.2 \%$ responde correctamente, llamando la atención que $33.3 \%$ de los estudiantes de Medicina Veterinaria responden negativamente, seguidos por los de Enfermería $(13.3 \%)$ y Gastronomía (6.7\%) ( $>00.05)$.

En la pregunta 9 sobre si el uso de guantes reemplaza la higiene de manos, solo el $82.2 \%$ del total de estudiantes responde adecuadamente, siendo esta una conducta primordial en la práctica profesional en las tres carreras. Por otro lado, la pregunta 7 sobre las uñas largas y la pregunta 8 sobre la relevancia del lavado de manos al inicio del turno o jornada fueron respondidas correctamente por el total de los estudiantes.

El 78\% de los estudiantes responden afirmativamente a las preguntas 14 y 15 , que tienen que ver con la relevancia intrínseca de la higiene de manos para evitar la transmisión de infecciones, mostrando una actitud adecuada y consciente sobre este acto de higienización. 
A la pregunta 16 ¿Realizaría higiene de manos con más frecuencia si mis compañeros/as me llamaran la atención por no hacer10 ?, $71 \%$ responde afirmativamente, entre ellos el total de estudiantes de Gastronomía $(\mathrm{p}<0.05)$, seguidos por Medicina Veterinaria (80\%) y Enfermería (33.3\%). Similar situación ocurre con la pregunta 17 ¿Realizaría higiene de manos con más frecuencia si mi comportamiento estuviera siendo observado y/o registrado por el supervisor?, donde $60 \%$ responde que Sí $(86.7 \%$ de Gastronomía $[\mathrm{p}<0.05]$ ), seguidos por Medicina Veterinaria [80\%] y Enfermería [33.3\%]). Se podría interpretar que un tercio de los estudiantes no tiene claro la motivación propia para realizar la higiene de manos.

Al considerar el tiempo como limitante para la ejecución del lavado de manos (pregunta 18), el 73.3\% de los estudiantes lo considera así, pero habiendo diferencia significativa entre las carreras (Enfermería [40\%] vs Gastronomía [93.3\%] y Medicina Veterinaria $[86.7 \%][p=0.002])$. En la pregunta 19 , ¿Transmito la importancia de la Higiene de Manos a mis compañeros/as y/o a estudiantes de otros cursos? solo $87 \%$ declara realizarlo, resaltando el compromiso del $100 \%$ de los estudiantes de Enfermería y de Gastronomía ( $\mathrm{p}=0.001$ ) al responder afirmativamente, a diferencia del $60 \%$ de los estudiantes de Medicina Veterinaria.

\section{Discusión}

La higiene de manos, a nivel mundial, es un procedimiento sencillo, rápido y económico que ayuda a prevenir las Infecciones Asociadas a Atención en Salud (IAAS), las Enfermedades Transmitidas por Alimentos (ETA) (Shama y Malik, 2013), así como el alto impacto económico que generan a los sistemas de salud pública (OMS, 2005), además de ayudar a prevenir las zoonosis (LloydSmith et al., 2009).
La OMS (2005) declara que la higiene de manos debe ser llevada a cabo con agua y jabón, pudiendo ser este corriente o antimicrobiano. En el presente estudio, solo $91 \%$ de los estudiantes indicaron haber recibido una adecuada formación en higiene de manos, siendo el mayor problema con los estudiantes de la carrera de Medicina Veterinaria; situación preocupante ya que se esperaba una repuesta afirmativa por el total de estudiantes a este sencillo acto, que es enseñado de manera formal en las carreras de educación superior y técnicas, que tienen relación con la salud humana, animal y/o alimentación (Feather et al., 2000; Stone 2001; Freeman et al., 2014). Otro resultado preocupante fue que los estudiantes, si bien presentan un buen nivel de conocimiento, tienen una inadecuada actitud frente a la higiene de manos. Feather et al. (2000) evidenciaron que el fracaso en la desinfección e higiene de manos en los médicos ad portas de obtener su título profesional, es reflejo de una deficiente actitud, comportamiento y convicción, y que es necesario incluir en los programas de formación académica la relevancia de la higiene de manos, así como procesos de evaluación para comprobar su aprendizaje.

Freeman et al. (2014), haciendo una revisión sistemática que incluyó 42 estudios de prevalencia sobre el lavado de manos, realiza una conclusión alarmante, al afirmar que a pesar de los conocidos efectos positivos de la higiene de manos en la salud, es escasamente realizada después del contacto con deposiciones, a nivel mundial. En el presente estudio también se pudo observar que los 45 estudiantes presentaban un adecuado nivel de conocimientos, pero que los resultados sobre la actitud frente al lavado de manos caen a un $70 \%$. Llama la atención que se le da mayor relevancia al lavado de manos cuando son observados, evaluados o cuando sus compañeros lo realizan. Esta actitud se condice con el estudio de Pittet et al. (2004), quienes concluyen que la adherencia se asocia con la conciencia de ser observado, la 
creencia de ser un modelo por seguir para otros colegas, comprobando además, que baja la adherencia cuando existe alta carga de trabajo. Esto ha llevado que algunos hospitales en Estados Unidos implementaran medidas como autoadhesivos en baños clínicos, donde solicitan a los pacientes preguntar al personal sanitario si se lavó las manos antes de atenderlos, e incluir esta conducta dentro de la medición de calidad en atención en los centros hospitalarios (Weinstein, 2004).

En este sentido, la metodología para evaluar la eficacia en la higiene de manos utilizada en el este estudio correspondió a la medición de ATP mediante la bioluminiscencia con el sistema de $3 \mathrm{M}^{\mathrm{TM}}$ Clean Trace ${ }^{\mathrm{TM}}$. Esta técnica mide los niveles de contaminación por materia orgánica como la contaminación microbiana (Nante et al., 2017), tanto en superficies como muestras de agua. Se basa en una reacción enzimática donde la Luciferina/Luciferasa en contacto con ATP genera luz, siendo directamente proporcional a la cantidad de ATP presente, y se expresa en unidades relativa de luz (URL) (Costa et $a l ., 2006)$. Esta técnica ha sido utilizada por la industria alimentaria hace más de 30 años, pero su uso en ambientes hospitalarios es limitado.

Según Carling (2013), la medición de ATP demuestra ser eficiente, pero tiene una baja especifidad (57\%), por lo que no es completamente recomendable como estándar en el ambiente hospitalario; sin embargo, reconoce que es de alto impacto en la formación de profesionales, dado lo inmediato de los resultados. Similar conclusión es reportada por Oliveira y Viana (2014), al afirmar que es un importante método para evaluar la limpieza de superficie, pero se requieren mas estudios para considerar este método en los servicios de salud. El Ministerio de Salud de Chile (MINSAL, 2019) en su documento «Recomendaciones para el aseo y desinfección de superficies para la prevención de IAAS» del año 2019, sugiere su uso según las normativas de cada centro de salud, de- pendiendo de la facilidad de uso y de la importancia de entregar retroalimentación a los supervisados.

Debido al alto impacto que genera esta metodología en la formación de los profesionales y teniendo como antecedentes la inadecuada actitud frente al lavado de manos, es que se decidió realizar la medición de bioluminiscencia denominada «antes lavado», mientras los estudiantes estuviesen realizando tareas en sus centros de prácticas. De esta manera, poder evidenciar el nivel de contaminación que pueden tener las manos, durante o posterior a un procedimiento, al manipular pacientes - humano o animal - así como alimentos y, demostrar cómo el lavado de manos es una medida efectiva en reducir esa contaminación, observada en la medición «después lavado». Se estima que los estudiantes, al ver cuantificado la contaminación de sus manos en URL y de manera inmediata, se generará un aprendizaje efectivo, donde el lavado de manos, adquiere la relevancia para que sea considerado un procedimiento esencial y casi automatizado, para la prevención en la transmisión de enfermedades y/o zoonosis.

Shama y Malik (2013) señalan que luego del uso de la bioluminiscencia como técnica de medición de limpieza se produce el «efecto Hawthrone», cuando el personal cambia su comportamiento, simplemente porque son conscientes de que su rendimiento está siendo monitoreado, pero que el cambio solo persiste durante la duración de la evaluación. En este sentido, Boyce et al. (2009) y Bustamante et al. (2018) concluyen que la medición de ATP mediante bioluminiscencia, entrega evidencia cuantitativa inmediata sobre la limpieza de superficie y lavado de mano clínico, respectivamente, apoyando los programas de intervención implementados. Por tanto, en cualquier método que apoye la evaluación de la higiene de manos en los servicios de salud, se deberia observar una mejora sustancial en la técnica de la higiene de manos así como una disminución en las infecciones nosocomiales (Pittet et al., 2000). 
Por todo lo anterior, se debe recalcar que la adecuada actitud y toma de conciencia sobre la relevancia de la correcta higiene de manos es fundamental para prevenir y disminuir las IAAS, ETAS y zoonosis, y en este sentido, el uso de la bioluminiscencia, por ser un método instantáneo y eficaz, puede ser utilizado como instrumento de evaluación para el apoyo durante la formación de los futuros profesionales relacionados al área de Una Salud, ya que favorece el cambio de conducta al demostrar la eficacia de la higienización en el momento.

\section{Agradecimiento}

Los autores agradecen a Gloria Bustos Fica, nutricionista, por su colaboración en el desarrollo de este proyecto.

\section{Literatura Citada}

1. Borges Primo MG, Ribeiro LCM, Da Silva Figueiredo LF, Albernaz Sirico SC, De Souza MA. 2010. Adesão à prática de higienização das mãos por profissionais de saúde de um Hospital Universitário. Rev Eletr Enf 12: 266-271, doi: 10.5216/ree.v12i2.7656

2. Boyce JM, Havill NL, Dumigan DG, Golebiewski M, Balogun O, Rizvani R. 2009. Monitoring the effectiveness of hospital cleaning practices by use of an adenosine triphosphate bioluminescence assay. Infect Control Hosp Epidemiol 30: 678-684. doi: 10.1086/598243

3. Bustamante F, Nuñez-Contreras J, Arellano-Villalon M, Crot W, Navarro P, Fuentes R. 2018. Bioluminiscencia: herramienta de medición y análisis en lavado de manos clínico aplicado a la odontología. Int J Odontostomat 12: 160-168. doi: $10.4067 /$ S0718-381X2018000200160

4. Carling P. 2013. Methods for assessing the adequacy of practice and improving room disinfection. Am J Infect Control 41: S20-S25. doi: 10.1016/j.ajic.2013.-01.003
5. Caparó EV. 2016. Validación de cuestionarios. Odontol Activa 1: 71-76. doi: 10.31984/oactiva.v1i3.200

6. Costa PD, Andrade NJ, Brandão SCC, Passos FJV, Soares NDFF. 2006. ATP-bioluminescence assay as an alternative for hygiene-monitoring procedures of stainless steel milk contact surfaces. Braz J Microbiol 37: 345-349. doi: 10.1590/S1517-83822006000300026

7. Feather A, Stone SP, Wessier A, Boursicot KA, Pratt C. 2000. 'Now please wash your hands': the handwashing behaviour of final MBBS candidates. J Hosp Infect 45: 62-64. doi: 10.1053/jhin.1999.0705

8. Fossum T. 2013. Small animal surgery. $4^{\text {th }}$ ed. St. Louis, Mo, USA: Elsevier Mosby. $1640 \mathrm{p}$.

9. Freeman MC, Stocks ME, Cumming $O$, Jeandron A, Higgins JPT, et al. 2014. Systematic review: Hygiene and health: systematic review of handwashing practices worldwide and update of health effects. Trop Med Int Healrh 19: 906-916. doi: 10.1111/tmi.12339

10. González-Cabrera J, FernándezPrada M, Trujillo Mendoza HM. 2012. Cuestionario para evaluar en médicos conductas, conocimientos y actitudes sobre la higiene de manos. Gac Sanit 26: 429-435. doi: 10.1016/ j.gaceta.2011.10.017

11. Lewis T, Griffith C, Gallo M, Weinbren M. 2008. A modified ATP benchmark for evaluating the cleaning of some hospital environmental surfaces. J Hosp Infect 69: 156-163. doi: 10.1016/j.jhin.2008.03.013

12. Lloyd-Smith JO, George D, Pepin KM, Pitzer VE, Pulliam JRC, et al. 2009. Epidemic dynamics at the humananimal interface. Science 326(5958): 1362-1367. doi: 10.1126/science. 1177345

13. [MINSAL] Ministerio de Salud. 2019. Circular C37 N¹0, Recomendaciones para el aseo y desinfección de superficies para la prevención de infecciones asociadas a la atención en salud (IASS). [Internet]. Disponible en: https://www. 
minsal.cl/wp-content/uploads/2019/03/ Circular-N-10-2019-Recomendaciones sobre-Aseo-y-Desinfeccio\%CC\%81nde-Superficies-Ambientales-para-laPrevencio\%CC\%81n-de-IAAS.pdf

14. Morán Cortés JF, Gimeno Benítez A, Martínez Redondo E, Sánchez Vega J. 2014. Conocimiento de la higiene de manos en estudiantes de enfermería. Enferm Glob 13: 136-147. doi: 10.6018/ eglobal.13.3.181501

15. Nante N, Ceriale E, Messina G, Lenzi D, Manzi P. 2017. Effectiveness of ATP bioluminescence to assess hospital cleaning: a review. J Prev Med Hyg 58: E177-E183.

16. Oliveira AC de, Viana REH. 2014. Adenosina trifosfato bioluminescência para avaliação da limpeza de superfícies: uma revisão integrativa. Rev Bras Enferm 67: 987-993. doi: 10.1590/00347167.2014670618

17. [OMS] Organización Mundial de la Salud. 2005. Directrices de la OMS sobre higiene de las manos en la atención sanitaria. OMS Ginebra. [Internet]. Disponible en: https://www.who.int/ patientsafety/information_centre/ Spanish_HH_Guidelines.pdf?ua $=1$

18. Pittet D, Allegranzi B, Sax H, Dharan S, Pessoa-Silva CL, Donaldson L, Boyce JM. 2006. Evidence-based model for hand transmission during patient care and the role of improved practices. Lancet Infect Dis 6: 641-652. doi: 10.1016/S1473-3099(06)70600-4

19. Pittet D, Hugonnet S, Harbarth S, Mourouga P, Sauvan V, Touveneau S, Perneger T V. 2000. Effectiveness of a hospital-wide programme to improve compliance with hand hygiene. Lancet
356: 1307-1312. doi: 10.1016/S01406736(00)02814-2

20. Pittet D, Simon A, Hugonnet S, Pessoa-Silva CL, Sauvan V, Perneger $\boldsymbol{T} \boldsymbol{V}$. 2004. Hand hygiene among physicians: performance, beliefs, and perceptions. Ann Intern Med 141: 1-8. doi: 10.7326/0003-4819-141-1-200407060-00008

21. Rey JSC, Prieto JR. 2015. Nivel de contaminación-descontaminación de la epidermis de las manos medida por luminiscencia. Enferm Dermatol 9: 34-45.

22. Seeger K, Griffiths MW. 1994. Adenosine triphosphate bioluminescence for hygiene monitoring in health care institutions. J Food Prot 57: 509-513. doi: 10.4315/0362-028X-57.6.509

23. Shama G, Malik DJ. 2013. The uses and abuses of rapid bioluminescencebased ATP assays. Int J Hyg Environ Health 216: 115-125. doi: 10.1016/j.ijheh.2012.03.009

24. Stone SP. 2001. Hand hygiene - the case for evidence-based education. J R Soc Med 94: 278-281. doi: 10.1177/ 014107680109400606

25. Weinstein RA. 2004. Hand hygiene of reason and ritual. Ann Intern Med 141: 65-66. doi: 10.7326/0003-4819-141-1200407060-00016

26. Weinstein RA, Milstone AM, Passaretti CL, Perl TM. 2008. Chlorhexidine: expanding the armamentarium for infection control and prevention. Clin Infect Dis 46: 274-281. doi: 10.1086/ 524736

27. [WHO] World Health Organization. 2013. Codex Alimentarius Commission: procedural manual. $21^{\text {st }} \mathrm{ed}$. Rome: Italy: WHO. 205 p. 